\section{The stimulus familiarization effect as a function of stimulus similarity ${ }^{1}$}

SUNNAN K. KUBOSE, University of North Carolina at Greensboro, Greensboro, N.C. 27412

Following a stimulus-familiarization phase, children responded in a button-pressing task to a familiarized stimulus (FS) and to three nonfamiliarized stimuli (NS1, NS2, NS3). The nonfamiliar stimuli varied in degree of similarity (color) to the FS; NS1 was the most similar and NS3 the least. A stimulus-familiarization effect (SFE) was obtained, with greater start speeds to NS3 than to FS. Analysis of start latencies produced a comparable effect (i.e., $F S>N S 3$ ) and also a "generalized" SFE (NS2>NS3). The results were interpreted within an OR-habituation hypothesis. The discrepancy between the speed and latency findings was discussed.

Several experiments (see Cantor, 1969) have involved use of a paradigm in which a stimulus is presented to a child $S$ repeatedly while he simply attends to the stimulus. Then, during a set of test trials, $S$ makes a simple motor response, half the time to the familiarized stimulus and half the time to a nonfamiliarized stimulus not previously presented. A stimulus familiarization effect (SFE) characterized by greater starting speeds to the nonfamiliarized than to the familiarized stimulus has been consistently obtained.

It has been suggested (Cantor, 1969) that the familiarization experience results in habituation of the "orientation reaction" (OR). ${ }^{2}$ According to the OR-habituation hypothesis, the initial occurrences of the stimulus in the familiarization phase elicit the $O R$ but that, with repeated presentations, the OR habituates to that stimulus. Thus, during the motor-task phase, the magnitude of the $O R$ should be greater to the nonfamiliarized than to the familiarized stimulus. Assuming that $S$ 's speed of responding in the motor task is positively related to the strength of the $O R$, responding to the nonfamiliarized stimulus should be faster than that to the familiarized stimulus.

The purpose of the present study was to provide evidence of some relevance to the OR-habituation formulation. Specifically, this investigation was an attempt to determine if the slower speed characterizing responses to a familiarized stimulus (as compared with responses to a physically dissimilar, nonfamiliarized stimulus) would also to some extent characterize responses to nonfamiliarized stimuli that are physically similar to the familiarized stimulus. The paradigm involved a familiarization phase, followed by a nondifferential reaction-time task in which a familiarization stimulus (FS) and three nonfamiliarized stimuli (NS1, NS2, NS3) each served as the signal to respond on a fourth of the trials. The nonfamiliar stimuli varied in degree of similarity (color) to the familiarized stimulus; NSI was the most similar and NS3 the least similar. The response measures included both start and travel speeds. For start speeds, the following rank order was predicted: NS3 $>$ NS2 $>$ NS1 $>$ FS. Such a result would be compatible with the supposition that $O R$ habituation occurs to the familiarized stimulus during the familiarization phase, and that the more similar the nonfamiliarized to the familiarized stimulus, the greater will be the extent of generalization of habituation to the nonfamiliarized stimulus.

Since the SFE is typically not obtained with execution speed measures, no significant differences were expected among travel speeds in the present study. METHOD

The Ss were 40 first-grade children (mean age: 6 years, 7 months) obtained from a public school in lowa City, Iowa.

The apparatus was a wooden box, 16 in. wide and $25 \mathrm{in}$. deep, painted flat black. The front of the apparatus consisted of two surfaces: (1) a horizontal surface, $3 \frac{1}{4}$ in. high, extending back $11 \frac{11 / 2}{\text { in., and }}$ (2) a vertical surface extending upward $11 \mathrm{in.} \mathrm{from} \mathrm{the} \mathrm{posterior} \mathrm{portion} \mathrm{of} \mathrm{the}$ horizontal surface. A circular opal glass aperture, 3 in. in diam, was located at the center of the vertical front surface. Four 6-W white bulbs were located within the box and behind the aperture. Colored photographic filters were placed between each of the bulbs and the aperture. One filter was red (Kodak Wratten Filter, No. 24), a second was orange (No. 22), a third was yellow (No. 102 and No. 15G), and a fourth was green (No. 58). The resulting four colored lights were approximately equated for apparent brightness by independent adult judges.

A black button and a white button, each in contact with microswitches, were placed 8 in. apart on the horizontal surface. A Hunter decade interval timer was used to control the duration of stimulus presentation during the familiarization phase and two Hunter KlocKounters were used for measuring response latencies in the reaction-time task.

Each $S$ was run individually in a dimly illuminated room. The $S$ was seated before the apparatus and told to watch carefully while a light came on and went off several times. For each S, 30 4-sec presentations were given. For half the Ss, the red light served as the familiarization stimulus; for the remaining Ss, the green light was presented. Within each of these two groups, there was an equal number of each sex. The E said "ready" before each stimulus presentation, using a restricted random order of 2- and 3-sec intervals between the ready signal and light onset. The intertrial interval was $5 \mathrm{sec}$.

Following the familiarization phase, $S$ was told to use the index finger of his preferred hand to hold down the black button. He was told that now one of several colored lights could appear. The S was then instructed to wait for the onset of the light, at which time he was to move his hand as quickly as possible to depress the white button. Following this, he was to return his hand to the black button. Each $S$ was given two practice trials in pushing the white button; no stimulus was presented for these two trials.

Forty-eight reaction-time trials were then administered. Each of the four different stimuli occurred once in each successive block of four trials, the order of occurrence within a block being determined randomly; there were four such random orders, assigned in equal numbers to the members of each counterbalancing group.

As in the familiarization phase, E said "ready" before each light presentation, using a restricted random order of $2-$ and 3-sec intervals between the ready signal and light onset. After each trial, E recorded S's response latencies (i.e., start time and travel time) to the nearest $.001 \mathrm{sec}$. Start time is the amount of time taken by the $S$ to lift his finger off the black button following stimulus onset. Travel time is the amount of time taken to press the white button after releasing the black start button.

\section{RESULTS}

A reciprocal transformation was used to change start and travel times to speed measures. S's mean speed of responding within four blocks of three trials each for both start and travel speed measures was determined separately for all four stimuli. Two mixed-design analyses of variance, one for each speed measure, were conducted. In each analysis, the within-Ss effects were: (1) stimulus type ${ }^{3}$ (FS vs NS1 vs NS2 vs NS3) and (2) trial blocks. The between-Ss 
effect was counterbalancing condition (red vs green familiarization stimulus).

The only significant effect obtained in the two analyses was that of trial blocks for starting speeds $(F=11.85, \mathrm{df}=3 / 114$, $\mathrm{p}<.001$ ). This effect is reflected in a general decrease in starting speeds across trial blocks. Although the main effect of stimulus type was not significant, the mean start speeds were ranked NS3 $>$ NS1 > NS2 $>$ FS. The mean start speeds for these stimulus types were $2.670,2.621,2.615$, and 2.586 , respectively, A preplanned comparison of NS3 vs FS was significant $(\mathrm{t}=2.44, \mathrm{df}=114, \mathrm{p}<.02)$.

Two additional analyses of variance of the same type were conducted on start and travel latencies. Significant main effects for trial blocks $(F=8.80, \quad d f=3 / 114$, $\mathrm{p}<.001)$ and stimulus type $(\mathrm{F}=2.88$, $\mathrm{df}=3 / 114, \mathrm{p}<.05$ ) were obtained in the start latency analysis. The mean start latencies for NS3, NS2, NS1, and FS were $.412, .435, .431$, and .439 , respectively. Orthogonal comparisons among the means revealed that the differences between NS3 and $F S(t=2.75, \mathrm{df}=114, \mathrm{p}<.01)$ and between NS3 and NS2 $(t=2.45, d f=114$, $p<.01)$ were significant. No significant effects were obtained in the travel latency analysis.

\section{DISCUSSION}

The following rank order of start speeds was predicted: NS3 $>$ NS2 $>$ NS1 $>$ FS. This prediction received partial support from the start-speed results. Although the main effect of stimulus type (FS vs NS1 vs NS2 vs NS3) was not significant, the mean start speeds were ranked NS3 $>$ NS1 $>$ NS2 > FS. A preplanned comparison of FS vs NS3 was significant, i.e., Ss responded significantly faster to NS3 than to FS. Thus, the SFE involving the familiarized stimulus and the most dissimilar nonfamiliarized stimulus was demonstrated.

For the start-latency analysis, a significant main effect for stimulus type was obtained. Furthermore, orthogonal comparisons among the means revealed that $S$ responded significantly faster to NS3 than to both FS and NS2. The difference between NS3 and NS2, which might be called a "generalized" SFE, indicates that the significantly slower responding to FS (compared with NS3) also characterized responding to a nonfamiliarized stimulus (NS2) which was similar to FS.

The finding of a significant main effect for stimulus type with start latencies and not with start speeds calls for some comment. In reaction-time tasks, there is a "ceiling" effect, i.e., a physical limit as to how fast $\mathrm{S}$ can respond. However, there is no "floor" effect, i.e., $S$ can respond as slowly as he chooses. This can cause the distribution of reaction-time latencies to be skewed. When this is the case, a reciprocal transformation tends to normalize the scores by decreasing the relative contribution of extremely long latencies to the overall means. Thus, when longer latencies tend to be associated with particular treatments or conditions (in the present case, with particular stimuli), one would expect the obtaining of a significant effect to be more likely for latency than for reciprocalized scores. 4

The effect of the transformation can also be seen when a comparison is made of the relative frequencies of $S s$ producing various stimulus rank orders for the speed and latency measures. For example, 31 out of $40 \mathrm{Ss}$ had smaller mean latencies for NS3 than for FS. However, with speed scores, only 26 out of $40 \mathrm{Ss}$ responded faster to NS3 than to FS (i.e., had greater mean speed scores for NS3 than for FS).

Although the present study does not provide a direct test of the OR-habituation hypothesis, the results appear to be consistent with such an interpretation. In addition to the OR-habituation explanation as discussed earlier, it is also necessary to assume that habituation of the OR generalizes to stimuli similar to the FS. Both Lynn (1966) and Thompson \& Spencer (1966) cite studies that have demonstrated generalization of OR-habituation, i.e., when a stimulus physically similar to the habituated stimulus is presented, the orientation reaction revives but is not as strong as that to the original stimulus. Since NS1, NS2, and NS3 represent stimuli of decreasing similarity to FS, one would expect less generalization of OR-habituation from FS to NS3 than from FS to either NS1 or NS2. In other words, FS elicits the weakest OR, NS3 elicits the strongest OR, while NS1 and NS2 elicit ORs of intermediate strength.

Several studies (Lansing, Schwartz, \& Lindsley, 1959; Maltzman \& Raskin, 1965; Meyers \& Joseph, 1968) have reported a positive relationship between the $O R$ and reaction time. Assuming that the strength of the OR is positively related to how fast Ss respond in the motor task of the present study, responding to NS3 should be faster than to FS, with responding to NS1 and
NS2 being faster than to FS but slower than to NS3. Overall, the results supported these expectations and were consistent with an OR-habituation hypothesis. This suggests that it might prove fruitful to conduct further studies investigating the OR-habituation hypothesis of the SFE. This hypothesis could be directly tested by employing independent measures (e.g., EEG, GSR) of the OR in a standard SFE paradigm.

\section{REFERENCES}

CANTOR, G. N. Effects of stimulus familiarization on child behavior. In J. P. Hill (Ed.), Minnesota symposia on child psychology. Vol. 3. Minneapolis: University of Minnesota Press (in press).

LANSING, R. W., SCHWARTZ, E., \& LINDSLEY, D. B. Reaction time and EEG activation under alerted and nonalerted conditions. Journal of Experimental Psychology, 1959, 53, 1-7.

LYNN, R. Attention arousal and the orientation reaction. New York: Pergamon Press, 1966.

MALTZMAN, 1., \& RASKIN, D. C. Effects of individual differences in the orienting reflex on conditioning and complex processes. Journal of Experimental Research in Personality, $1965,1,1-16$.

MEYERS, W. J., \& JOSEPH, L. J. Response speed as related to CS-prefamiliarization and GSR-responsivity. Journal of Experimental Psychology, 1968, 78, 375-381.

SOKOLOV, E. N. Perception and the conditioned reflex. New York: Pergamon Press, 1963.

THOMPSON, R. F., \& SPENCER, W. A. Habituation: A model phenomenon for the study of neuronal substrates of behavior. Psychological Review, 1966, 73, 16-43.

\section{NOTES}

1. This article is based on an MA Equivalence thesis presented to the Institute of Child Behavior and Development, University of lowa. The advice and assistance of Prof. Gordon N. Cantor are gratefully acknowledged. The author also wishes to express his appreciation to James Blank (Director of Elementary Education, Jowa City Community School District) and Jerome Hogarty (Principal, Henry Sabin Elementary School).

2. Writers on this topic (e.g., Sokolov, 1963; Lynn, 1966) conceive of the OR as involving a constellation of behavioral and physiological responses, including head tuming, eye movements, pupil dilation, EEG changes, peripheral vasoconstriction, cephalic vasodilation, and the GSR.

3. For the group familiarized on red, orange served as NS1, yellow as NS2, and green as NS3. For the group familiarized on green, yellow served as NS1, orange as NS2, and red as NS3.

4. It should be mentioned that the frequency distributions of latency scores for the treatment groups in the present study did not appear to be markedly skewed. Therefore, use of the latency scores did not seem to constitute a violation of the normality assumption. 